Portland State University

PDXScholar

7-1-1996

\title{
Scattering Properties of Dense Media from Monte Carlo Simulations with Application to Active Remote Sensing of Snow
}

Lisa M. Zurk

Portland State University

L. Tsang

Dale P. Winebrenner

Follow this and additional works at: https://pdxscholar.library.pdx.edu/ece_fac

Part of the Electrical and Computer Engineering Commons

Let us know how access to this document benefits you.

\section{Citation Details}

Zurk, L. M., Tsang, L., \& Winebrenner, D. P. (1996). Scattering properties of dense media from Monte Carlo simulations with application to active remote sensing of snow. Radio Science, 31(4), 803-819.

This Article is brought to you for free and open access. It has been accepted for inclusion in Electrical and Computer Engineering Faculty Publications and Presentations by an authorized administrator of PDXScholar. Please contact us if we can make this document more accessible: pdxscholar@pdx.edu. 


\title{
Scattering properties of dense media from Monte Carlo simulations with application to active
}

\section{remote sensing of snow}

\author{
L. M. Zurk ${ }^{1}$ \\ Applied Physics Laboratory, University of Washington, Seattle \\ L. Tsang \\ Electrical Engineering Department, University of Washington, Seattle \\ D. P. Winebrenner \\ Applied Physics Laboratory, University of Washington, Seattle
}

\begin{abstract}
Monte Carlo simulations are used to derive the phase matrix, effective permittivity, and scattering coefficient for a random medium consisting of densely packed spheres up to 5000 in number. The results include correlated scattering and coherent wave interaction among the scatterers. The Monte Carlo simulations are based on a multiple-scattering formulation of the Foldy-Lax equations. It is shown that the derived phase matrix is in good agreement with dense media radiative transfer theory for copolarized scattering. The depolarization, however, can be substantially larger than conventional theory. Two methods are used to analyze the behavior of the coherent wave to obtain the real part of the effective permittivity. For the small particle case both methods yield values of permittivity that agree with the results of mixing formulas such as the Clausius-Mossoti mixing formula. The phase matrix and scattering coefficient obtained by simulation are used in a second-order radiative transfer model to predict the amount of backscatter from a layer of snow. It is also shown that sticky spheres, which can be used to model metamorphosed snow, produce high levels of copolarized and depolarized backscatter that can exceed the independent scattering model.
\end{abstract}

\section{Introduction}

An important feature of a discrete random medium with densely packed scatterers (i.e., scatterers that occupy more than a few percent by volume) is that the correlation of the particles' positions affects the scattering physics and invalidates the independent scattering as-

\footnotetext{
${ }^{1}$ Now at Massachusetts Institute of Technology Lincoln Laboratory, Lexington.

Copyright 1996 by the American Geophysical Union.

Paper number 96RS00939.

0048-6604/96/96RS-00939\$11.00
}

sumption. This has been shown experimentally [Ishimaru and Kuga, 1982; Koh, 1992]. To account for the correlation between particles, analytic dense media theory, such as the quasi-crystalline approximation (QCA) [Tsang and Kong, 1980, 1982] and quasi-crystalline approximation with coherent potential (QCA-CP) [Tsang and Kong, 1980; Tsang et al., 1985], has been assumed for the first moment of the field. In early work the QCA approximation was applied with the hole correction to obtain a set of equations for the average field in an ensemble of scatterers [Varadan et al., 1979; Varadan and Varadan, 1980]. More recently, the Percus- 
Yevick pair distribution function was used with QCA to calculate the extinction coefficient and produced good agreement with controlled laboratory experiments [Mandt et al., 1992; West et al., 1994]. Because of the advent of modern computers and efficient computational methods, Monte Carlo simulations by direct solution of Maxwell's equations are receiving necessary attention. Both rough surface problems [Pak et al., 1995] and discrete scatterer problems [Tsang et al., 1992; Zurk et al., 1995] have been solved in this manner. However, the final results of bistatic scattering are calculated differently between rough surface simulations and dense media simulations. In rough surface scattering one directly solves Maxwell's equations to calculate the bistatic scattering coefficients. In dense media simulations, because of computer limitations, one uses a few thousand particles to perform Monte Carlo simulations to simulate the extinction coefficient, phase matrix, and absorption coefficient. These quantities are then used in radiative transfer theory to calculate the bistatic scattering properties of the dense media. Monte Carlo simulations have been used to calculate the extinction rates using up to 5000 spheres. The numerical procedure is based on the multiple scattering equations of Foldy-Lax using vector spherical waves as a basis [Tsang et al., 1992; Lu et al., 1995]. The results include coherent wave interaction among the spheres. The extinction rates derived from the simulations are in excellent agreement with QCA-CP.

To calculate intensities, analytic theory has been applied for the second moment of the field leading to the Bethe-Salpeter equation. A correlated ladder approximation has been applied to the Bethe-Salpeter equation. It has been shown that QCA-CP together with the correlated ladder approximation obeys energy conservation. The two approximations together also lead to the dense media radiative transfer equation (DMRT). In the long wavelength limit, the phase matrix of the dense media radiative transfer equation is of the form of the Rayleigh phase matrix but with different extinction coefficients and albedos from conventional radiative transfer theory of independent scattering.

In this paper we employ Monte Carlo simulations to calculate the phase matrix and the effective permittivity using up to 5000 dielectric spheres. Numerical results indicate that the copolarized parts of the phase matrix are in good agreement with those of dense media radiative transfer theory under QCA-CP and the correlated ladder approximation. However, the depolarized component can be substantially higher. The strong depolarized return is due to coherent wave interaction among the spheres leading to electrical dipole moments of spheres that are not parallel to the incident electric field. The level of depolarization is still substantially lower than that of the copolarized component. Thus it has little effect in the energy conservation that QCA-CP and the correlated ladder approximation obey.

We next apply results to microwave remote sensing of snow-covered regions [Tsang and Kong, 1992; Kuga et al., 1991]. The scattering coefficient and the phase matrix derived from the Monte Carlo simulations are used in a secondorder radiative transfer model [Shin and Kong, 1981] to determine the amount of scattering from a snow layer overlying a homogeneous halfspace of soil. These results are compared to those obtained using DMRT and the independent scattering assumption. The characteristics of a snow layer can also change because of metamorphic forces [Colbeck, 1982] and affect the microwave response. In previous work [Ding et al., 1994] we introduced the use of a sticky particle pair function which can be used to model the clustering of the snow grains and in the work of Zurk et al. [1995] we presented extinction and absorption coefficient from sticky particles. In this paper we show that the sticky particle model used in a radiative transfer model can match simultaneously the copolarized and depolarized microwave scatter return of snow.

Another attribute of a discrete random medium is its effective permittivity. In this paper we compare the response of the coherent wave with- 
in the Monte Carlo volume to that of a homogeneous volume to determine the effective permittivity. We also directly obtain the real part of the permittivity by tracking the amplitude and phase of the average internal electric dipole as the coherent wave travels through the medium. The results of the effective permittivity are in good agreement with Clausius-Mossoti.

\section{Radiative Transfer Equation}

The transport of radiant energy through a space of randomly distributed spherical particles, as shown in Figure 1, can be expressed by the vector radiative transfer (RT) equation for the specific intensity $\mathbf{I}$ :

$$
\begin{aligned}
\cos (\theta) & \frac{d \mathbf{I}(\theta, \phi, z)}{d z}=-\kappa_{e} \mathbf{I}(\theta, \phi, z) \\
& +\int_{0}^{2 \pi} d \phi^{\prime} \int_{0} \pi d \theta^{\prime} \sin \left(\theta^{\prime}\right) \mathbf{P}\left(\theta, \phi, \theta^{\prime}, \phi^{\prime}\right) \\
& \cdot \mathbf{I}(\theta, \phi, z)
\end{aligned}
$$

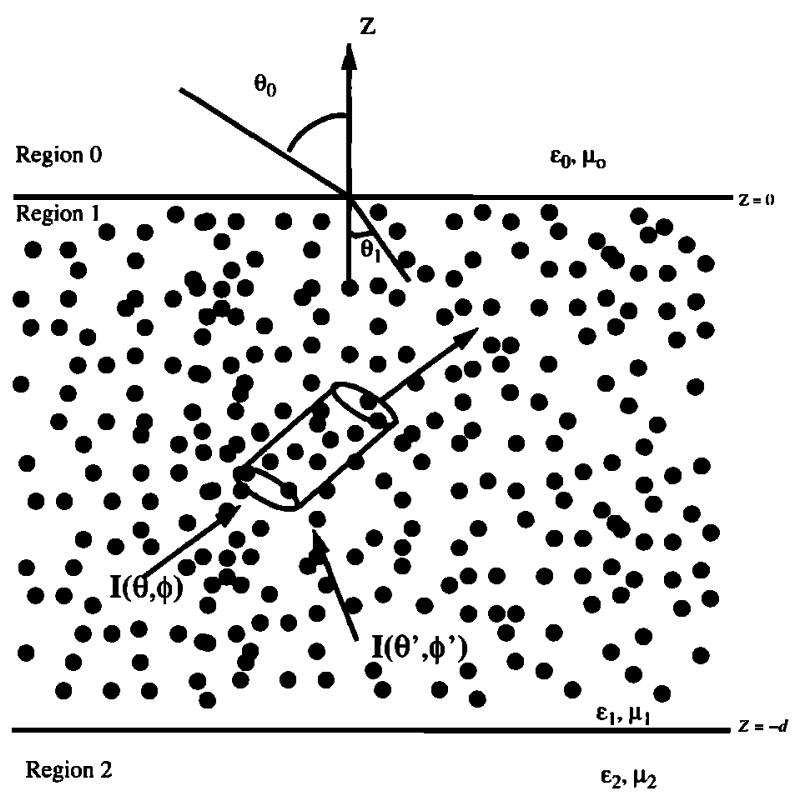

Figure 1. Geometry of the radiative transfer problem for a planar layer of spherical scatters in region 1 covering a homogeneous half-space in region 2 where $Z$ is normal to the planar interfaces. Elemental volume for calculation of radiative transfer parameters shown. where $\kappa_{e}$ is the extinction coefficient with $\kappa_{e}=$ $\kappa_{s}+\kappa_{a}, \kappa_{s}$ and $\kappa_{a}$ are the scattering and absorption coefficients, respectively, and $\mathbf{P}\left(\theta, \phi, \theta^{\prime}, \phi^{\prime}\right)$ is the phase matrix describing redistribution of scattered energy from direction $\theta, \phi$ into direction $\theta^{\prime}, \phi^{\prime}$. Application of boundary conditions at the planar interfaces $z=0$ and $z=-d$ requires knowledge of the effective permittivity $\epsilon_{\text {eff }}$ in region 1 . Thus solution of (1) requires specification of $\kappa_{e}, \mathbf{P}\left(\theta, \phi, \theta^{\prime}, \phi^{\prime}\right)$, and $\epsilon_{\text {eff }}$ and will be influenced by the method with which they are obtained.

To calculate the radiative transfer parameters, we consider an elemental volume which contains many particles, as shown in Figure 1. The derivation of the transfer equation is based on radiative energy in and out of the volume element. Note that there is not just one particle in the elemental volume but there are many particles.

In the following sections we discuss three different methods of calculating the extinction coefficients and the phase matrix. The methods are (1) classical scattering assumption, (2) DMRT, and (3) Monte Carlo simulation.

\subsection{Classical Radiative Transfer}

The classical assumption for scattering in a collection of randomly distributed spherical scatterers is that of independent scattering. Under this assumption the particles in the elemental volume scatter and absorb independently. Thus if there are $N$ particles in the elemental volume, the extinction cross section of all the particles is

$$
\sigma_{e}^{N}=N \sigma_{e}
$$

where $\sigma_{e}$ is the extinction cross section of one particle. The extinction coefficient is defined as the extinction cross section per unit volume. Thus if $V$ is the volume of the elemental volume, then

$$
\kappa_{e}^{\text {ind }}=\frac{N}{V} \sigma_{e}=n_{0} \sigma_{e},
$$

where $n_{0}$ is the number of particles per unit volume. 
Similarly, for a single particle the distribution of scattered energy is given by the Stokes matrix $\sigma$. Under the independent scattering assumption the phase matrix is the average of the Stokes matrix over the distribution of particles, and for identical particles

$$
\mathbf{P}\left(\theta, \phi, \theta^{\prime}, \phi^{\prime}\right)=n_{0} \sigma\left(\theta, \phi, \theta^{\prime}, \phi^{\prime}\right) .
$$

For small scatterers this reduces to the Rayleigh phase matrix [Tsang et al., 1985].

\subsection{DMRT}

The assumption of independent scattering ignores correlated wave interaction when the scatterers are densely packed. For dense random media the effective wavenumber $K$ can be calculated using the quasi-crystalline approximation with coherent potential [Tsang et al., 1985]. In the low frequency limit the dispersion relation is

$$
\begin{aligned}
K^{2}= & k^{2}+f_{v}\left(k_{S}^{2}-k^{2}\right) / \gamma \\
& \cdot\left(1+i \frac{2}{9} K a^{3}\left(k_{s}^{2}-k^{2}\right) / \gamma\right. \\
& \left.\cdot\left\{1+4 \pi n_{0} \int_{0}^{\infty} d r r^{2}[g(r)-1]\right\}\right) \\
\gamma= & 1+\left(k_{s}^{2}-k^{2}\right)\left(1-f_{v}\right) /\left(3 K^{2}\right)
\end{aligned}
$$

where $k_{s}$ is the wavenumber of the scatterers, $k$ is the wavenumber of the background, $a$ is the radius of the spheres, $f_{v}$ is the fractional volume of the scatterers, and $g(r)$ is the pair distribution function. The albedo $\tilde{\omega}=\kappa_{s} / \kappa_{e}$ can be derived from the second moment as

$$
\begin{aligned}
\tilde{\omega}= & \frac{1}{2 K^{\prime \prime}} \frac{f_{v} a^{3}}{3}\left|k_{s}^{2}-k^{2}\right|^{2} n_{0} \\
& \cdot\left\{1+4 \pi n_{0} \int_{0}^{\infty} d r r^{2}[g(r)-1]\right\},
\end{aligned}
$$

where $K^{\prime \prime}$ is the imaginary part of the effective wavenumber and $\kappa_{e}=2 K^{\prime \prime}$. As noted previously, DMRT follows from QCA-CP and the correlated ladder approximation. In the long wavelength limit the phase matrix of the dense media radiative transfer equation is of the form of the Rayleigh phase matrix but with the scattering coefficient calculated by the above relationships.

\subsection{Simulation}

As an alternative to either the classical model or DMRT, Monte Carlo simulations can be used to directly determine the extinction coefficient, the phase matrix, and the effective permittivity. These are calculated by using a large number of scatterers in the elemental volume and taking into account their coherent wave interactions to determine the collective scattering and interaction of the $N$ particles in the elemental volume. Of course, $N$ has to be a large number and convergence with respect to $N$ has to be tested. For example, the extinction coefficient can be calculated by considering the transfer of energy within this volume from the coherent wave into the incoherent wave. The elemental volume must satisfy three criteria. First, the volume must be small enough that the attenuation of energy in the incident wave as it travels through cube is small compared to the incident energy. Second, the volume must be large enough relative to the wavelength so that the phase of the wave varies across the volume and creates random phase situations. Finally, the number of scatterers enclosed within the volume must be large enough to represent a random sampling of scatterers.

Calculation of the radiative transfer parameters from Monte Carlo simulation differs from the previous approaches in that the $N$ particle collective behavior is considered [West et al., 1994]. The extinction coefficient from this volume is derived from the $N$ particle cross section per unit volume. The phase matrix is obtained from the $N$ particle bistatic cross section per unit volume. The effective permittivity is determined from calculation of the coherent scattered field. The phase and amplitude from the elemental volume is compared with a homogeneous volume to ascertain the effective permittivity. The details of these calculations are described in the following two sections.

\section{Monte Carlo Simulations}

The wave propagation and scattering through a large number of scatterers is computed using 
an exact formulation based on Maxwell's equations. The advantage of this method is that exact values for the extinction coefficient and phase matrix can be obtained and then compared with those obtained from dense media theory. In this section we discuss how the scattering and absorption coefficients and the phase matrix can be computed from the scattered incoherent fields. We then discuss the case of spherical scatterers, where the formulation of the multiple scattering equations is in terms of vector spherical wave functions, and present the iterative solution technique for the Monte Carlo simulations.

\subsection{Scattering and Absorption Coefficients}

The scattering and absorption of radiant energy from the incident wave represents transfer of energy from the coherent beam into incoherent power due to the presence of the scatterers. To separate the coherent and incoherent components, the scattered field is averaged to give the coherent scattered field

$$
<\mathbf{E}_{s}>=\frac{1}{N_{r}} \sum_{\sigma=1}^{N_{r}} \mathbf{E}_{s}^{\sigma}
$$

where $\sigma$ is the realization index with $\sigma=1,2, \ldots$, $N_{r}$ realizations. $\mathbf{E}_{s}^{\sigma}$ is the final scattered field from the elemental volume of many scatterers and includes their coherent near- and intermediate-range interactions. The incoherent field is the difference between the total field and the coherent field $\mathcal{E}_{s}^{\sigma}=\mathbf{E}_{s}^{\sigma}-<\mathbf{E}_{s}>$. Calculation of the scattering coefficient $\kappa_{s}$ from the incoherent scattered field $\mathcal{E}_{s}$ can be expressed as

$$
\kappa_{s}=\frac{1}{V} \int_{0}^{\pi} d \theta_{s} \sin \left(\theta_{s}\right) \int_{0}^{2 \pi} d \phi_{s} \frac{R^{2}}{N_{r}} \sum_{\sigma=1}^{N_{r}}\left|\mathcal{E}_{s}^{\sigma}\right|^{2}
$$

The absorption coefficient is due to absorption within the particle

$$
\sigma_{a N}=\sum_{\beta=1}^{N} \int_{V} k_{s} \epsilon_{r}^{\prime \prime}\left|\mathbf{E}^{\beta}\left(\mathbf{r}^{\prime}\right)\right|^{2} d V^{\prime}
$$

where the integration is over the sphere volume $V$ and $\epsilon_{r}^{\prime \prime}$ is the imaginary part of the relative permittivity for the spheres and $\mathbf{E}_{\text {int }}$ is the internal electric field.

\subsection{Calculation of the Phase Matrix}

An $N$ particle collective scattering amplitude $\mathcal{F}_{\alpha \beta}$ can be defined for a volume element where $N$ is a large number. The subscripts $\alpha=1$ and 2 and $\beta=1$ and 2 designate the polarization of the incident and scattered waves, respectively, such that $\alpha, \beta=1$ describes a wave polarized perpendicular to the plane of incidence and $\alpha, \beta=2$ is a wave polarized parallel to the plane of incidence. To determine an expression for $\mathcal{F}_{\alpha \beta}$, we can use the definition of the incoherent electric field $\mathcal{E}_{s}^{\sigma}=\mathbf{E}_{s}^{\sigma}-<\mathbf{E}_{s}>$ to write

$$
\begin{gathered}
\mathcal{E}_{\alpha \beta}=\frac{e^{i k r}}{r} \mathcal{F}_{\alpha \beta} \\
\mathcal{F}_{\alpha \beta} \equiv\left[F_{\alpha \beta}-<F_{\alpha \beta}>\right],
\end{gathered}
$$

where $F_{\alpha \beta}$ is the scattering amplitude of the total scattered electric field $\mathbf{E}_{s}$. Multiplying by a complex conjugate and taking the ensemble average then gives

$$
<\mathcal{E}_{\alpha \beta} \mathcal{E}_{\alpha^{\prime} \beta^{\prime}}^{*}>=\frac{1}{r^{2}}<\mathcal{F}_{\alpha \beta} \mathcal{F}_{\alpha^{\prime} \beta^{\prime}}>
$$

where for $\alpha=\alpha^{\prime}$ and $\beta=\beta^{\prime}$.

$$
<\left|\mathcal{F}_{\alpha \beta}\right|^{2}>=<\left|F_{\alpha \beta}\right|^{2}>-\left|<F_{\alpha \beta}>\right|^{2} .
$$

In the Monte Carlo simulations the incident wave travels in the $\hat{z}$ direction and is polarized in the $\hat{y}$ direction (Figure 2). Thus $\mathcal{F}_{11}$ and $\mathcal{F}_{21}$ can be determined by considering the two components of the scattered field that travels in the $x, z$ plane or with $\phi_{s}=0$. Likewise, $\mathcal{F}_{22}$ and $\mathcal{F}_{12}$ represent scattering from an incident wave with parallel polarization and can be determined by consideration of the scattered wave traveling in the $y, z$ plane with $\phi_{s}=\pi / 2$. For example, 


$$
\begin{aligned}
\left\langle\left|\mathcal{F}_{11}\right|^{2}\right\rangle= & \frac{R^{2}}{N_{r}} \sum_{\sigma=1}^{N_{r}}\left|\mathcal{E}_{h s}^{\sigma}\left(\phi_{s}=0, \theta_{s}\right)\right|^{2} \\
<\left(\mathcal{F}_{11} \mathcal{F}_{12}^{*}\right)>= & \frac{R^{2}}{N_{r}} \sum_{\sigma=1}^{N_{r}} \mathcal{E}_{h s}^{\sigma}\left(\phi_{s}=0, \theta_{s}\right)(15) \\
& \cdot \mathcal{E}_{h s}^{\sigma *}\left(\phi_{s}=\pi / 2, \theta_{s}\right),
\end{aligned}
$$

where $\mathcal{E}_{h s}\left(\phi_{s}=0, \theta_{s}\right)^{\sigma}$ is the horizontal component of the incoherent scattered field of the $\sigma$ th realization evaluated at $\phi_{s}=0$ and for $0 \leq \theta_{s} \leq 2 \pi$. The phase matrix in the plane of incidence, $\mathbf{P}_{\mathrm{poi}}$, is of the form of the Stokes matrix (see, for example, Tsang et al. [1985], p. 133) but with the single particle scattering functions $f_{\alpha \beta}$ replaced by the $N$ particle collective scattering amplitudes $\left\langle\mathcal{F}_{\alpha \beta}\right\rangle$, which are functions of the angle between the incident and scattered wave, $\Theta$.

The full electromagnetic phase matrix over all input and output angles can be obtained by transformation of $\mathbf{P}_{\text {poi }}$ through rotation angles $\phi_{1}$ and $\phi_{2}$. The rotation angles are determined by the direction of the vertically polarized wave vector with respect to the normal $\hat{n}$ to the plane of incidence. In terms of the incident and scattered angles they are

$$
\begin{aligned}
\sin \phi_{1} & =\left(\hat{v}_{i} \times \hat{n}\right) \cdot \hat{k}_{i} \\
& =\left[\hat{v}_{i} \times\left(\hat{k}_{i} \times \hat{k}_{s}\right) / \sin \Theta\right] \cdot \hat{k}_{i} \\
& \left.=\hat{v}_{i} \cdot \hat{k}_{s} \hat{v}_{s}\right) \cdot \hat{k}_{s} \\
\sin \phi_{2} & =\left[\hat{v}_{s} \times\left(\hat{k}_{i} \times \hat{k}_{s}\right) / \sin \Theta\right] \cdot \hat{k}_{s} \\
& =[\hat{v} \\
& =\hat{v}_{s} \cdot \hat{k}_{i} .
\end{aligned}
$$

The phase matrix $\mathbf{P}$ is then given by

$$
\mathbf{P}\left(\theta, \phi, \theta^{\prime}, \phi^{\prime}\right)=\mathbf{L}\left(-\phi_{2}\right) \mathbf{P}_{\text {poi }} \mathbf{L}\left(\phi_{1}\right)
$$

where $\mathbf{L}$ is a the rotation matrix given by [Ishimaru, 1978]

$$
\mathbf{E}(\phi)=\left[\begin{array}{cccc}
\cos ^{2} \phi & \sin ^{2} \phi & \frac{1}{2} \sin 2 \phi & 0 \\
\sin ^{2} \phi & \cos ^{2} \phi & -\frac{1}{2} \sin 2 \phi & 0 \\
-\sin 2 \phi & \sin 2 \phi & \cos 2 \phi & 0 \\
0 & 0 & 0 & 1
\end{array}\right]
$$

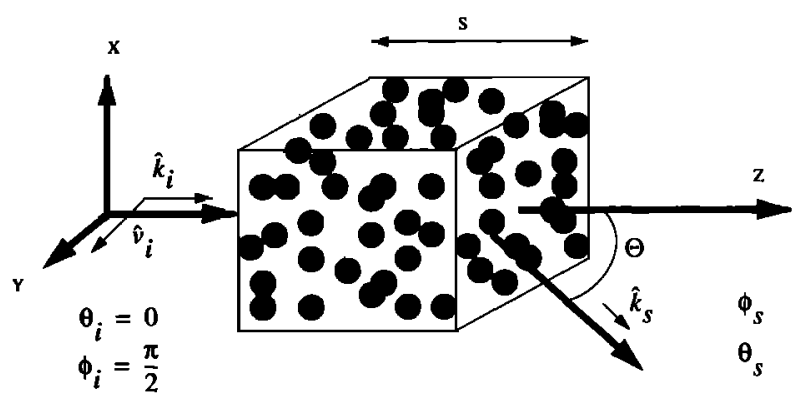

Figure 2. Monte Carlo test volume shown with incident electric field traveling in the $\hat{k}_{\imath}=\hat{z}$ direction and polarized in the $\hat{v}_{i}=\hat{y}$ direction. The cubic test volume has a side of length $s$ and volume $v=s^{3}$. The scattered fields are in the direction $\theta_{s}$ and $\phi_{s}$ and $\Theta$ is the angle between $\hat{k}_{i}$ and $\hat{k}_{s}$.

\subsection{Configuration for Monte Carlo Simulations for Spherical Scatterers}

The configuration for the Monte Carlo simulations is shown in Figure 2. The behavior of the incoherent wave in the Monte Carlo simulations is considered on a per unit volume basis using an elemental volume of Figure 2 and is independent of the shape of the volume. In each realization, $N$ spheres with permittivity $\epsilon_{s}$ and radius $a$ are randomly deposited [Zurk et al., 1995] into the elemental box of volume $V=s^{3}$ or sphere with $V=(4 \pi / 3) s^{3}$.

Quantities that are appropriate for use in the solution of the radiative transfer equation represent statistical averages with respect to the scatterer placement. They are obtained from Monte Carlo simulations by averaging over realizations where each realization consists of a different sphere distribution within the test volume $V$. The statistics of the sphere locations can be depicted with the bivariate probability measure called the pair distribution function. For random deposition of nonoverlapping spheres the pair distribution function corresponds to the Percus-Yevick pair distribution function. An alternate deposition method for sticky hard spheres [Ding et al., 1994; Zurk et al., 1995] utilizes a parameter $\tau$ which governs the potential of spheres to cluster together. Smaller values of $\tau$ result in more clustering or "sticking" of the 
spheres and can cause a pronounced increase in scattering.

For all of the simulation results presented in this paper a single orientation of the incident wave was used and is shown in Figure 2. The relationship between the incident field vectors and the angles $\theta_{i}$ and $\phi_{i}$ is

$$
\begin{gathered}
\hat{k}_{i}=\hat{x} \sin \theta_{i} \cos \phi_{i}+\hat{y} \sin \theta_{i} \sin \phi_{i}+\hat{z} \cos \theta_{i} \\
\hat{v}_{i}=\hat{x} \cos \theta_{i} \cos \phi_{i}+\hat{y} \sin \theta_{i} \sin \phi_{i}-\hat{z} \sin \theta_{i} \\
\hat{h}_{i}=-\hat{x} \sin \phi_{i}+\hat{y} \cos \phi_{i} .
\end{gathered}
$$

The incident wave is an electric field traveling in the $\hat{k}_{i}=\hat{z}$ direction and polarized in the $\hat{v}_{i}=$ $\hat{y}$ direction, with $\theta_{i}=0$ and $\phi_{i}=\pi / 2$. The scattered field has components as given above with $\theta_{i}$ replaced by $\theta_{s}$ and $\phi_{i}$ by $\phi_{s}$.

\subsection{Solution of Multiple-Scattering Equations}

Unlike continuous random media, discrete scatterers have well-defined boundaries. Since the space occupied by the random scatterers and the space occupied by the background are distinguishable, with each region having its own permittivity, the fields in each region can be expressed in a complete vector spherical wave expansion and equated at the scatterer boundaries using Maxwell equations. For the case of discrete scatterers in a homogeneous background, Maxwell equations can be cast into the FoldyLax multiple-scattering equations in matrix notation as [Tsang et al., 1985]

$$
\begin{aligned}
\mathbf{a}^{\mathbf{s}(\alpha)} & =\sum_{\substack{\beta=1 \\
\beta \neq \alpha}}^{N} \mathbf{T}^{\alpha} \sigma\left(k \mathbf{r}_{\alpha} \mathbf{r}_{\beta}\right) \mathbf{a}^{s(\beta)} \\
& +\exp \left(i \mathbf{k}_{\mathbf{i}} \cdot \mathbf{r}_{\alpha}\right) \mathbf{T}^{\alpha} \mathbf{a}_{\text {inc }}
\end{aligned}
$$

where $\mathbf{a}^{s(\alpha)}$ is the vector of coefficients for spherical wave harmonics of the multiple scattered field for particle $\alpha, \mathbf{a}_{\text {inc }}$ is the coefficient of the incident wave, $k$ is the wavenumber of the background media, $\mathbf{k}_{i}$ is the wavenumber of the incident wave, $N$ is the number of spheres in the containing volume, $\boldsymbol{\sigma}\left(k \mathbf{r}_{\alpha} \mathbf{r}_{\beta}\right)$ is the vector spherical wave transformation matrix, $\mathbf{T}^{\alpha}$ is the $T$ matrix for scatterer $\alpha$, and $\mathbf{r}_{\alpha}$ and $\mathbf{r}_{\beta}$ are the centers of particles $\alpha$ and $\beta$, respectively. Note that (23) does not contain a far-field approximation and includes near- and intermediate-range interactions.

The final scattered field $\mathbf{E}_{s}$ from $N$ spheres at an observation point $r$ is

$$
\begin{aligned}
\mathbf{E}_{\mathrm{s}(\mathrm{r})} & =\sum_{m n}\left[a_{m n}^{s(M)} \mathbf{M}_{\mathrm{mn}}(k r, \theta, \phi)\right. \\
& \left.+a_{m n}^{s(N)} \mathbf{N}_{\mathrm{mn}}(\mathrm{kr}, \theta, \phi)\right]
\end{aligned}
$$

where $\mathbf{M}_{m n}$ and $\mathbf{N}_{m n}$ are outgoing vector spherical wave functions [Tsang et al., 1985].

Equation (23) can be interpreted physically as follows. The final scattered field coefficients for a particle $\alpha$ depend on the excitation experienced by that particle. This excitation is due to both the original incident field $a_{i n c}$ as well as the radiated field from surrounding particles $\mathbf{a}^{s(\beta)}$. The scattered field from other particles can be translated from those particles to particle $\alpha$ using Huygen's equivalency principle through use of the vector spherical wave transformation matrix $\sigma\left(k \mathbf{r}_{\alpha} \mathbf{r}_{\beta}\right)$. Finally, the response of $\alpha$ to the excitation it experiences is specified by its $T$ matrix $\mathbf{T}^{\alpha}$.

The internal electric field appearing in (10) can also be written as a sum of spherical wave functions as

$$
\begin{aligned}
\mathbf{E}_{\mathrm{int}}(r) & =\sum_{m n}\left[c_{m n}^{(M)} R g \mathbf{M}_{m n}(k r, \theta, \phi)\right. \\
& \left.+c_{m n}^{(N)} R g \mathbf{N}_{m n}(k r, \theta, \phi)\right]
\end{aligned}
$$

The relationship between the scattered field coefficients $a_{m n}^{s}$ and the internal field coefficients $c_{m n}$ is given by [Tsang et al., 1985; Zurk et al., 1995]

$$
\mathbf{c}=\left(-R g \mathbf{Q}^{t}\right)^{-1} \mathbf{a}^{s} .
$$

For an $N$ particle system there are $N$ equations of the form of (23) which must be solved simultaneously. In the iterative approach the initial solution is the response of the scatterer 
to the incident field in the absence of other scatterers. Each successive iteration pertains to a higher order of scattering. We continue to iterate (23) until the maximum change in the field coefficients is less than $5 \%$, at which point the solution is considered to have converged. The number of iterations necessary to achieve convergence is a direct indicator of the strength of the multiple scattering between particles and depends on fractional volume, dielectric contrast, and sphere radius.

\section{Coherent Wave Calculations}

In the following sections we present calculations based on the behavior of the coherent wave within the Monte Carlo volume. These calculations are used to determine (1) the behavior of internal dipole moments within the scatterers and (2) the real part of the effective permittivity for the random medium.

\subsection{Dipole Orientation}

The electric dipole for the $j$ th sphere as calculated in Monte Carlo simulations can be written as

$$
\mathbf{p}_{\text {int }}^{j}=\int_{V}\left(\epsilon_{s}-\epsilon_{0}\right) \mathbf{E}_{\text {int }}(\mathbf{r}) d \mathbf{V},
$$

where $\mathbf{E}_{\text {int }}(\mathbf{r})$ is given by (25) in terms of the vector spherical wave functions. The wave functions can be converted to Cartesian coordinates and the integration performed analytically.

Under the QCA approximation the dipole moment can be written as

$$
\begin{gathered}
\mathbf{p}_{\mathrm{int}}^{\mathrm{QCA}}=\frac{3 v_{0} \epsilon_{0} y}{1-f_{v} y} E_{0} e^{i \mathbf{K}_{r} \cdot \mathbf{r}} \hat{e} \\
y=\frac{\epsilon_{s}-\epsilon_{0}}{\epsilon_{s}+2 \epsilon_{0}},
\end{gathered}
$$

where $v_{0}$ is the volume of the sphere and $K_{r}$ is the real part of the effective wavenumber whose calculation will be discussed later.

The incident electric field will cause alignment of the internal dipoles, and this alignment will oscillate with the incident frequency. However, there will also be slight misalignment in each particle's dipole due to the effect of the scattered fields from nearby particles. To isolate random deviations of the dipole moment, the phase dependency of the coherent wave needs to be removed. This will be referred to as "dephasing" and can be achieved by multiplication of $\mathbf{p}_{\text {int }}^{j}$ in (26) for sphere $j$ by the phase factor $\exp \left(-i \mathbf{K}_{r} \cdot \mathbf{r}_{j}\right)$.

The coherent addition of the dipole moments will be utilized in section 5.2 and is defined as follows. Define the reference point $\mathbf{r}_{c}$ as the center of the Monte Carlo test volume (for this calculation we enhance spherical symmetry by using a test volume which is spherical in shape). We can then define the coherent dipole sum $\mathbf{P}_{\text {sum }}\left(\alpha_{l}\right)$ as the addition of the dephased dipoles of spheres within a distance $\alpha_{l}$ of the reference point:

$$
\mathbf{p}_{\text {sum }}\left(\alpha_{l}\right)=\frac{1}{N_{r}} \sum_{1}^{N_{r}} \frac{1}{N_{l}} \sum_{1}^{N_{l}} \mathbf{p}_{\text {int }}^{j} e^{-i \mathbf{K}_{r} \cdot \mathbf{r}_{j}}
$$

where $N_{l}$ is the number of spheres $j$ that satisfy $\left|\mathbf{r}_{j}-\mathbf{r}_{c}\right| \leq \alpha_{l}$. The coherent sum can be written $p_{\text {sum }}\left(\alpha_{l}\right)=p_{x}\left(\alpha_{1}\right) \hat{x}+p_{y}\left(\alpha_{l}\right) \hat{y}+p_{z}\left(\alpha_{l}\right) \hat{z}$.

\subsection{Effective Permittivity}

The idea of an effective permittivity for a random medium is that if the random medium were replaced by a homogeneous medium that responds to electromagnetic excitation in an identical fashion as the original random medium, the permittivity of the homogeneous medium is said to be the effective permittivity of the random medium. This concept has been explored and utilized extensively in the past.

In the Monte Carlo simulations the coherent wave responds to the size, shape, and effective permittivity of the test volume. To determine the effective permittivity of the collection of spherical scatterers, the scattered coherent field can be compared with the field scattered from a homogeneous volume of the same size and shape as the Monte Carlo test volume. The permittivity of this homogeneous volume when the scat- 
tered fields are the same is the effective permittivity of the random medium.

The test volume used in the Monte Carlo simulations for the effective permittivity calculations is a sphere with diameter $s$. The Mie scattering cross section from a sphere with the same diameter and with a permittivity $\epsilon_{\text {eff }}$ is denoted as $\sigma_{s, \mathrm{Mie}}$. The permittivity at which the Mie cross section is equal to that produced by the coherent scattering from the Monte Carlo spherical test volume is then, by definition, the effective permittivity of the random media. This quantity can be determined by varying $\epsilon_{\text {eff }}$ in the calculation of $\sigma_{s, \text { Mie }}$ until the following equality is satisfied:

$\sigma_{s, \mathrm{Mie}}=\int_{0}^{\pi} d \theta_{s} \sin \left(\theta_{s}\right) \int_{0}^{2 \pi} d \phi_{s} \frac{R^{2}}{N_{r}} \sum_{\sigma=1}^{N_{r}}\left|\mathbf{E}_{s}^{\sigma}\right|^{2}$

where $\mathbf{E}_{s}^{\sigma}$ is the total scattered field. The effective permittivity of the Mie sphere contains an imaginary component that accounts for the loss of energy due to scattering within the medium. The imaginary part of the effective permittivity can be calculated from its relationship to the extinction coefficient under the assumption that $\epsilon_{\text {eff }}^{\prime \prime} \ll \epsilon_{\text {eff }}^{\prime}$ :

$$
\begin{aligned}
& \kappa_{e}=2 k_{0} \operatorname{Im}\left\{\sqrt{\epsilon_{\mathrm{eff}}}\right\} \\
&=2 k_{0} \operatorname{Im}\left\{\sqrt{\epsilon_{\mathrm{eff}}^{\prime}} \sqrt{1+i \frac{\epsilon_{\mathrm{eff}}^{\prime \prime}}{\epsilon_{\mathrm{eff}}^{\prime}}}\right\} \\
& \simeq k_{0} \frac{\epsilon_{\text {eff }}^{\prime \prime}}{\sqrt{\epsilon_{\text {eff }}^{\prime}}} \\
& \epsilon_{\text {eff }}^{\prime \prime}=\frac{\kappa_{e} \sqrt{\epsilon_{\text {eff }}^{\prime}}}{k_{0}}
\end{aligned}
$$

where $\epsilon_{\text {eff }}^{\prime}$ and $\epsilon_{\text {eff }}^{\prime \prime}$ are the real and imaginary parts of the effective dielectric constant, respectively.

\section{Results}

In this section we present results from Monte Carlo simulations. Section 5.1 discusses the na- ture of the phase matrix obtained from simulation and section 5.2 gives some physical understanding of the source of the observed depolarization. Section 5.3 presents the effective permittivity of the random media and comparisons with Clausius-Mossoti and QCA-CP. Section 5.4 presents the backscatter from a layer of snow and comparisons of results of independent scattering, DMRT, and Monte Carlo simulations.

\subsection{Phase Matrix from Monte Carlo Simulations}

The phase matrix was calculated as given in section 3.2 for spheres with $k a=0.2$, permittivity $\epsilon_{s}=3.2 \epsilon_{0}$, and a fractional volume of $35 \%$. Values for the scattering coefficients can be found in Table 1. The independent scattering assumption grossly overestimates the scattering at this fractional volume. QCA-CP predicts scattering rates that are lower than those calculated with Monte Carlo simulations. This discrepancy could be due to the low-frequency solution to QCA-CP.

The elements of the upper corner of the phase matrix (i.e., $\left|\mathcal{F}_{11}\right|^{2},\left|\mathcal{F}_{21}\right|^{2},\left|\mathcal{F}_{12}\right|^{2},\left|\mathcal{F}_{22}\right|^{2}$ ) are shown in Figures 3 and 4 as functions of $\Theta$. The solid curve corresponds to nonsticky particles and the dash-dotted curve is for particles deposited in the test volume with an adhesive potential of $\tau=0.05$. The open circles are

Table 1. Scattering Coefficient for Spheres With $a=0.56 \mathrm{~mm}$ at $35 \%$ Fractional Volume and $\epsilon_{\mathrm{s}}=$ $3.2 \epsilon_{0}$

\begin{tabular}{lc}
\hline Simulation & $\begin{array}{c}\kappa_{s}, \\
\times 10^{-3} / \mathrm{cm}\end{array}$ \\
\hline $\begin{array}{l}\text { Independent } \\
\text { Quasi-crystalline approximation }\end{array}$ & 3.36 \\
$\begin{array}{l}\text { Quasi-crystalline approximation, } \\
\text { with coherent potential }\end{array}$ & 0.23 \\
$\begin{array}{l}\text { Monte Carlo } \\
\text { Monte Carlo, } \\
\tau=0.38\end{array}$ & 0.54 \\
$\begin{array}{c}\text { Monte Carlo, } \\
\tau=0.05\end{array}$ & 0.87 \\
$\begin{array}{c}\text { Monte Carlo, } \\
\tau=0.01\end{array}$ & 1.17 \\
\hline
\end{tabular}



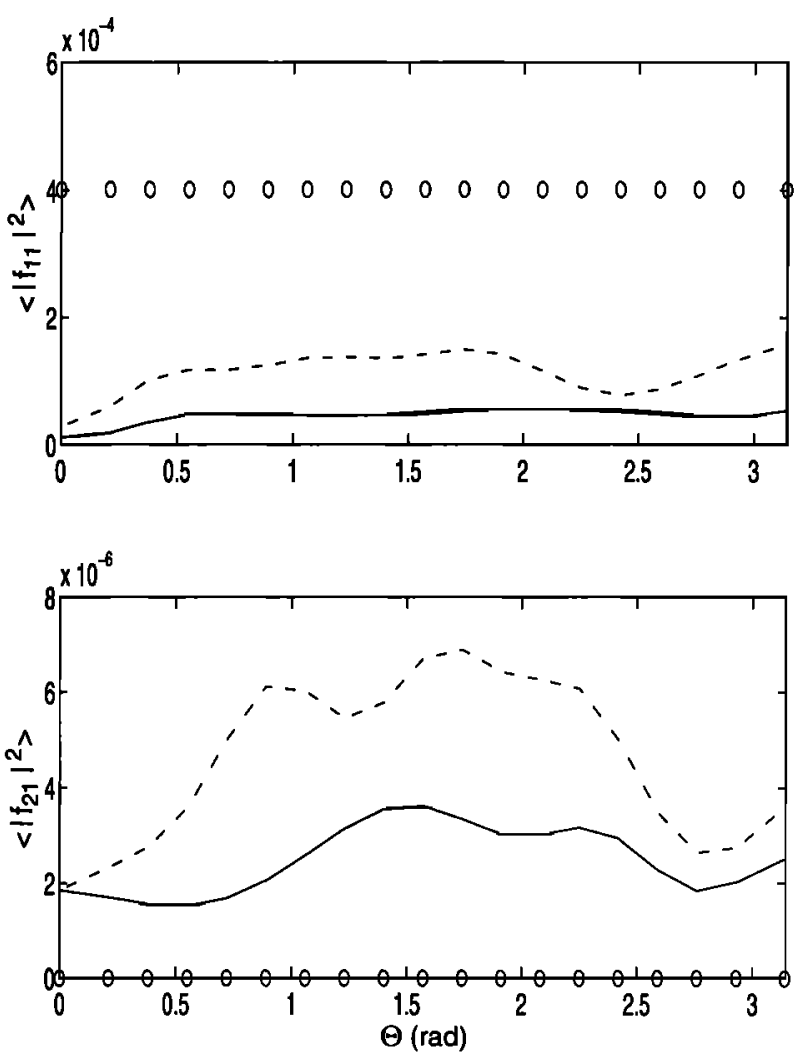

Figure 3. Elements of the phase matrix for an incident wave polarized perpendicular to the plane of incidence as a function of $\Theta$. Results for spheres with $\epsilon_{s}=3.2 \epsilon_{0}, 35 \%$ fractional volume, and $a=0.6 \mathrm{~mm}$ at $16 \mathrm{GHz}$. Monte Carlo results shown for nonsticky spheres (solid curve) and sticky spheres with $\tau=0.05$ (dash-dotted curve). Independent scattering shown as open circles. Top panel shows $\left\langle\left|\mathcal{F}_{11}\right|^{2}>\right.$. Bottom panel shows $\left\langle\left|\mathcal{F}_{21}\right|^{2}\right\rangle$.

the Rayleigh phase matrix elements where the scattering coefficient is computed with the independent scattering assumption. Depolarization from spheroids with an axial ratio of $c / a=1.70$ is shown as crosses in Figure 4.

Several characteristics of the Monte Carlo phase matrix can be immediately noticed. The copolarized intensities $\left(\left|\mathcal{F}_{11}\right|^{2}\right.$ and $\left.\left|\mathcal{F}_{22}\right|^{2}\right)$ have the same angular dependency as the Rayleigh matrix, but the overall intensity is lower because of the more realistic scattering coefficient. As discussed by Zurk et al. [1995], the sticky particles have scattering levels that are much greater than the nonsticky particles because of the effec- tively larger particle size. For spherical particles the off-diagonal terms $\left(\left|\mathcal{F}_{12}\right|^{2}\right.$ and $\left.\left|\mathcal{F}_{21}\right|^{2}\right)$ of the Rayleigh matrix are zero. Monte Carlo simulations of both sticky and nonsticky particles give nonzero depolarized intensities that are approximately 2 orders of magnitude lower than the copolarized intensities. The level of depolarization calculated in the Monte Carlo simulations for the nonsticky spheres is comparable to the depolarization resulting from spheroids with axial ratios of 1.70 .

Figures 5 and 6 are plots of the depolarization to copolarization ratios $\left(\int_{\Theta} \sigma_{21} / \int_{\Theta} \sigma_{11}\right.$ and $\left.\int_{\Theta} \sigma_{12} / \int_{\Theta} \sigma_{22}\right)$ as a function of permittivity and
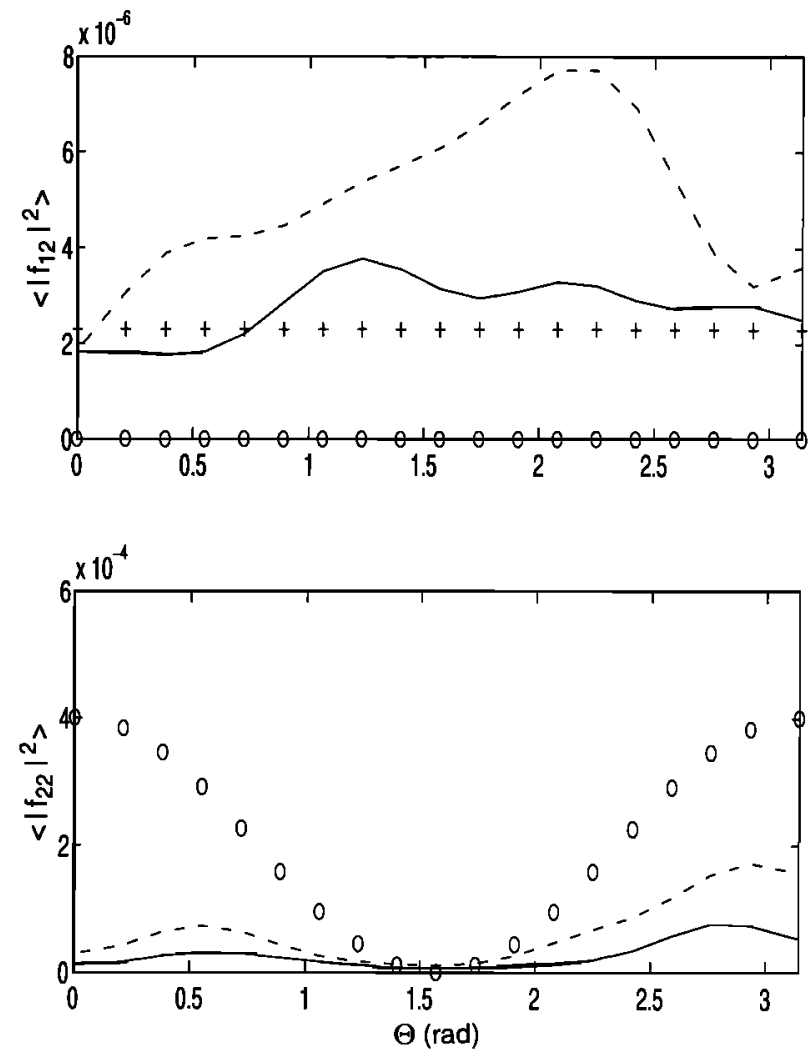

Figure 4. Elements of the phase matrix for an incident wave polarized parallel to the plane of incidence as a function of $\Theta$; parameter values the same as Figure 3. Monte Carlo results shown for nonsticky spheres (solid curve) and sticky spheres with $\tau=0.05$ (dash-dotted curve). Independent scattering shown as open circles and spheroids with $c / a=1.70$ shown as crosses. Top panel shows $\left\langle\left|\mathcal{F}_{12}\right|^{2}\right\rangle$. Bottom panel shows $<\left|\mathcal{F}_{22}\right|^{2}>$. 


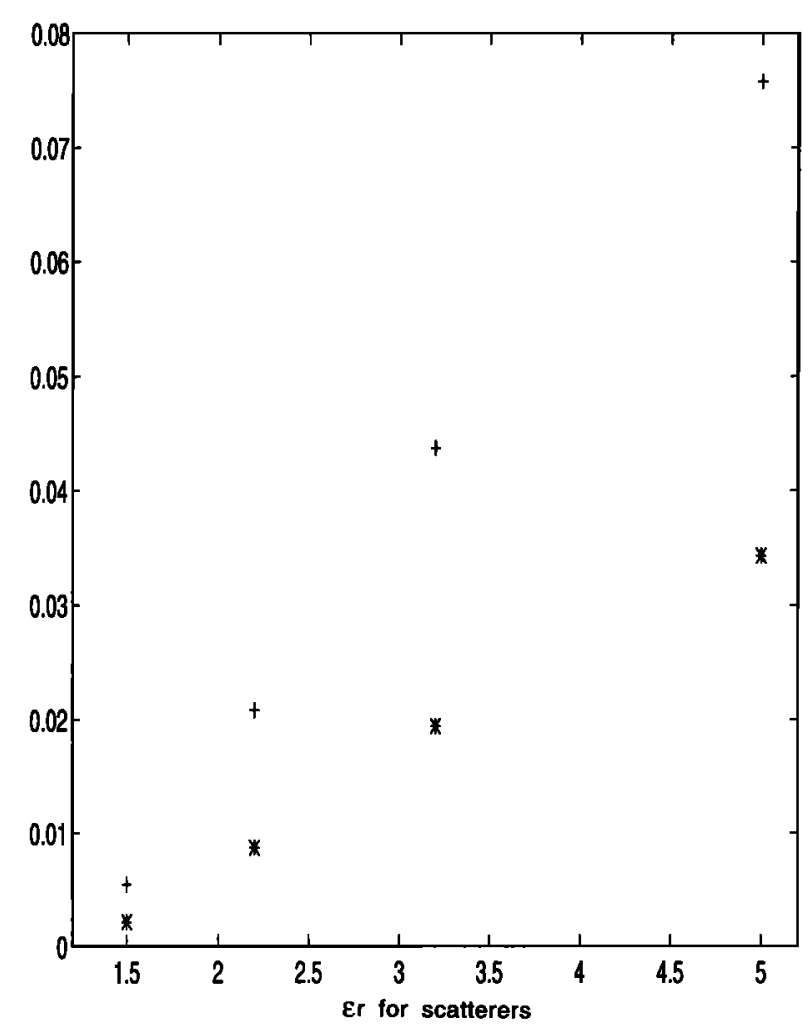

Figure 5. Depolarization level $\sigma_{21} / \sigma_{11}$ (asterisks) and $\sigma_{12} / \sigma_{22}$ (crosses) as a function of the spheres' relative permittivity $\epsilon_{s} / \epsilon_{0}$.

fractional volume, respectively. The ratio is larger for parallel polarization because of the lack of copolarized energy as $\Theta$ approaches $\pi / 2$.

\subsection{New Depolarization Versus Classical}

For a single sphere in the presence of plane wave excitation the dipole moment within the sphere is exactly aligned with the incident polarization, and the scattered field in the scattering plane will not undergo any depolarization. Since in classical radiative transfer theory the phase matrix depends only on single-particle scattering quantities, the phase matrix contains zero depolarization components in the plane of incidence. The reason for this thinking was the belief that the effect of any small misalignment of a sphere's internal dipole (relative to the incident field) due to the presence of scattered fields from surrounding spheres will cancel. This cancella- tion is due to the random, isotropic nature of the relative locations of the spheres, which causes a randomness in the perturbation of the dipole orientation. For the case of a large enough number of spheres the net depolarization was thought to be zero.

A central assumption of this idea is that the wave will continue to add the dipole moments coherently over a large enough distance for this cancellation to occur. If the distance required is large in comparison to the electromagnetic wavelength, the wave will decorrelate and the dipole moments separated by wavelengths apart will not be added coherently. In other words, there is an intrinsic length scale over which coherent addition of dipole radiation is achieved by the electromagnetic wave. If there are enough random, isotropically positioned spheres within this length scale, there will be no depolarization.

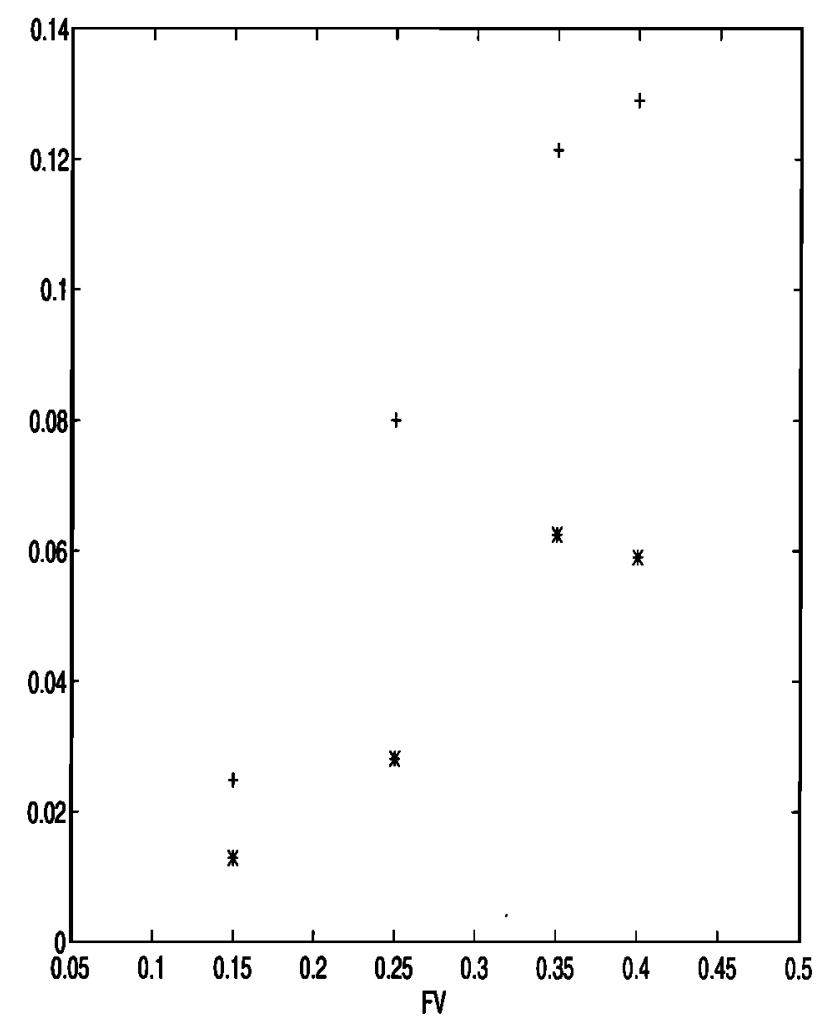

Figure 6. Depolarization level $\sigma_{21} / \sigma_{11}$ (asterisks) and $\sigma_{12} / \sigma_{22}$ (crosses) as a function of fractional volume. 
If, however, only a few hundred spheres are encountered before the wave decorrelates, then the cross-dipole moment will not be zero. If we define the length scale for the correlated addition of dipoles as $\lambda_{\text {eff }}$, then the number of spheres contained within a volume $\lambda_{\text {eff }}^{3}$ must be large for the cross dipole to go to zero.

In an attempt to quantify the concept of a length scale for the electromagnetic wave we used (29) to calculate explicitly the orientation of the internal electric dipole for 2500 spheres with fractional volume of $20 \%$ and permittivities of $3.2 \epsilon_{0}$ and $2.2 \epsilon_{0}$. The magnitude of $p_{x}$ and $p_{z}$ relative to $\left|\mathbf{p}_{\text {sum }}\right|$ (i.e., those components misaligned with the incident $y$ polarization) for $\epsilon_{s}=3.2 \epsilon_{0}$ is shown in a $\log$ scale
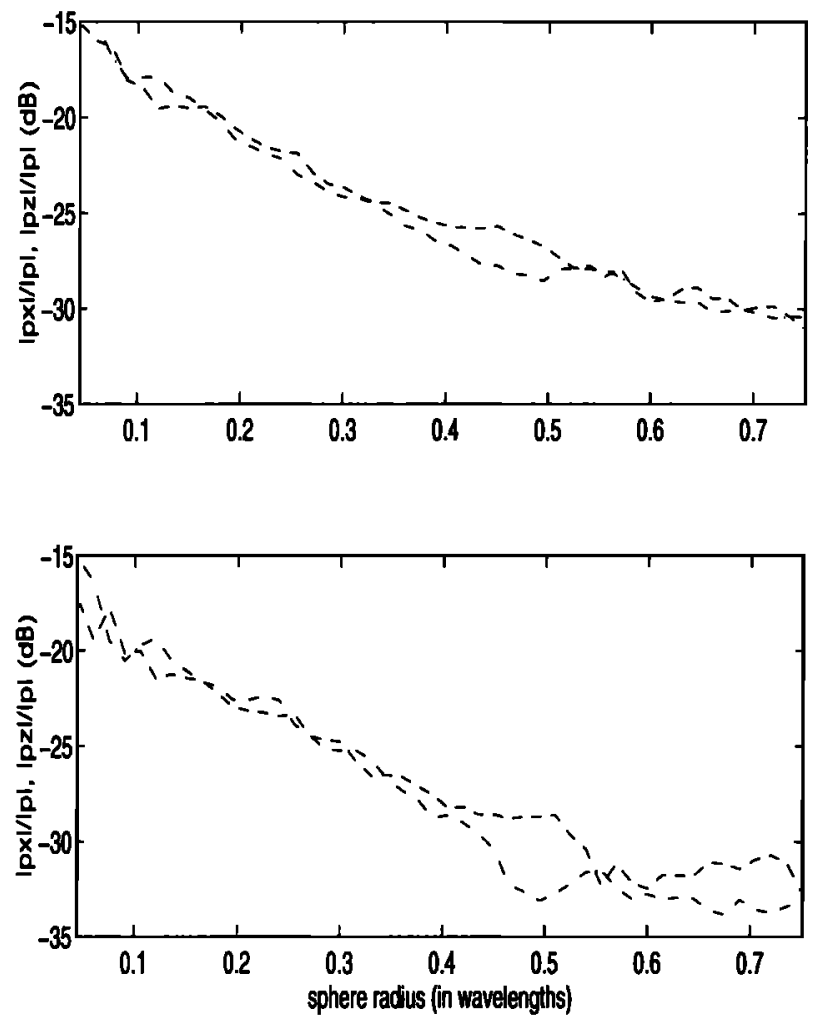

Figure 7. Magnitude of the $x$ (dot-dashed curve) and $z$ (dashed curve) components of the internal dipole normalized to $\left|p_{y}\right|$ and computed as specified in (29). Results shown for $20 \%$ fractional volume with 2500 spheres for (top) $\epsilon_{s}=3.2 \epsilon_{0}$ and (bottom) $\epsilon_{s}=2.2 \epsilon_{0}$. The $x$ axis is the radius $\alpha_{l} / \lambda_{0}$ of the spherical volume over which the sum is computed. as a function of $\alpha_{l} / \lambda_{0}$ in the top of Figure 7 . The figure shows that the level of the depolarization decreases as more and more spheres are introduced into the sum in (29). The resultant net depolarization from the Monte Carlo simulations of $-17 \mathrm{~dB}$ in the phase matrix implies that the electromagnetic wave only adds coherently within the spherical region of diameter $d_{l}=2 \alpha_{l}=0.27 \lambda_{0}$. For a permittivity of $2.2 \epsilon_{0}$ (shown in the bottom of Figure 7) the depolarization drops more quickly because of the diminished influence of the scattered fields relative to the incident excitation. The depolarization from the simulations corresponds to coherent addition within a distance of $d_{l}=0.36 \lambda_{0}$. The effective wavelengths for $\epsilon_{s}=3.2 \epsilon_{0}$ and $\epsilon_{s}=2.2 \epsilon_{0}$ are $\lambda_{\text {eff }}=0.88 \lambda_{0}$ and $\lambda_{\text {eff }}=0.92 \lambda_{0}$, respectively, where the effective media calculation is described in the next section. These distances indicate that the electromagnetic wave adds the dipoles coherently over roughly a quarter of an effective wavelength. Any existing depolarization within $\sim \frac{1}{4} \lambda_{\text {eff }}$ will be added coherently and incoherently outside that distance. This concept has important relevance to the distance scale over which coherent averaging is to be performed.

\subsection{Effective Media Calculations}

As outlined in section 4.2, the coherent scattering from the Monte Carlo volume can be used to calculate the effective permittivity of a collection of particles within the volume. This calculation was tested by comparing the coherent field scattering pattern as a function of angle from cubes of lengths $s=1.27, s=1.39$, and $s=1.50$ containing 3000,4000 , and 5000 spheres, respectively, of permittivity $\epsilon=3.2 \epsilon_{0}$ at $20 \%$ fractional volume. Since the size of the Monte Carlo volume varied, the coherent scattering pattern did as well. Scattering from the three cubes was then calculated with the Born approximation and an effective permittivity with the real part ranging between $\epsilon_{\mathrm{eff}}=1.1$ and $\epsilon_{\mathrm{eff}}=1.35$. For all three sized cubes at an effective permittivity of $\epsilon_{\text {eff }}=1.27$ the magnitude of the forward scat- 
tering peak and the fine structure of the sidelobes agreed well with that obtained from the Monte Carlo simulations. For higher fractional volumes a spherical test volume was used for the Monte Carlo simulations, and the coherent scattering was calculated exactly from the Mie scattering. Figure 8 shows the real part of the effective permittivity as a function of fractional volume. Also shown is the permittivity calculated from the Clausius-Mossoti mixing formula and the quasi-crystalline approximation with coherent potential. It is interesting to note that the Monte Carlo results agree quite well with those obtained from the mixing formula but are slightly below the values predicted by QCA-CP. In recent work [Zurk et al., 1995] we showed that the extinction rates calculated from Monte Carlo simulations agreed with those produced

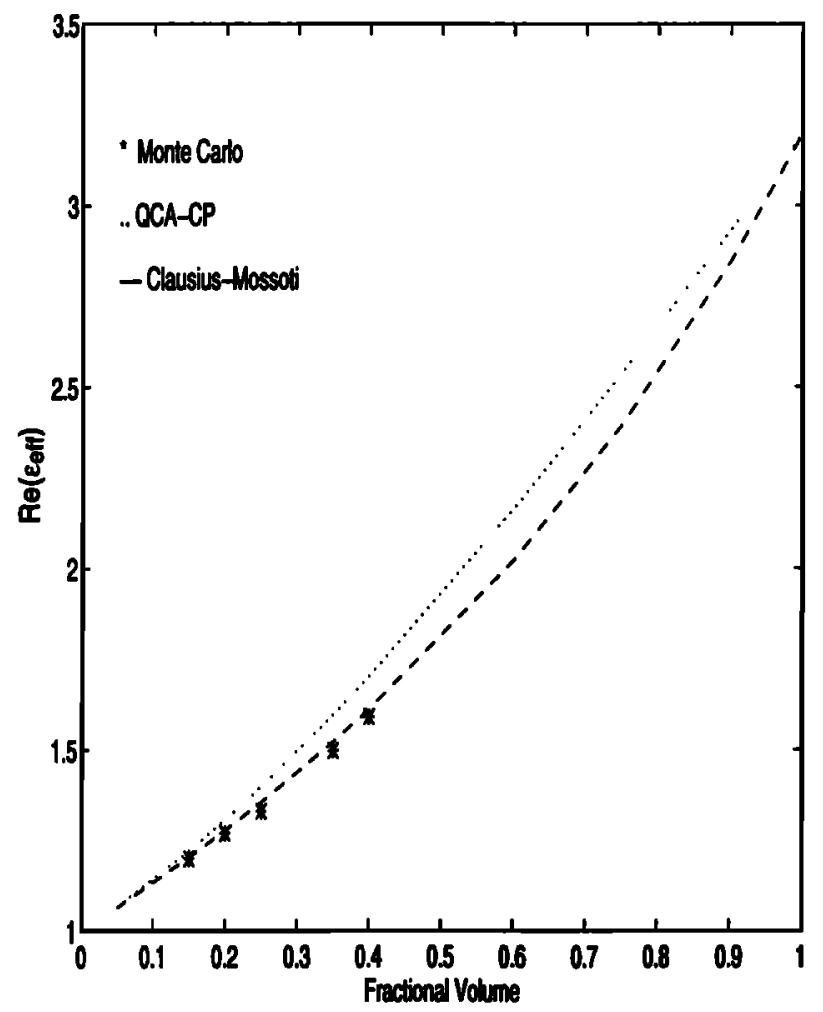

Figure 8. Real part of the effective permittivity $\epsilon_{\text {eff }}$ versus fractional volume calculated from Monte Carlo simulations (asterisks) and compared with Clausius Mossotti (dashed curve) and QCA-CP (dotted curve).

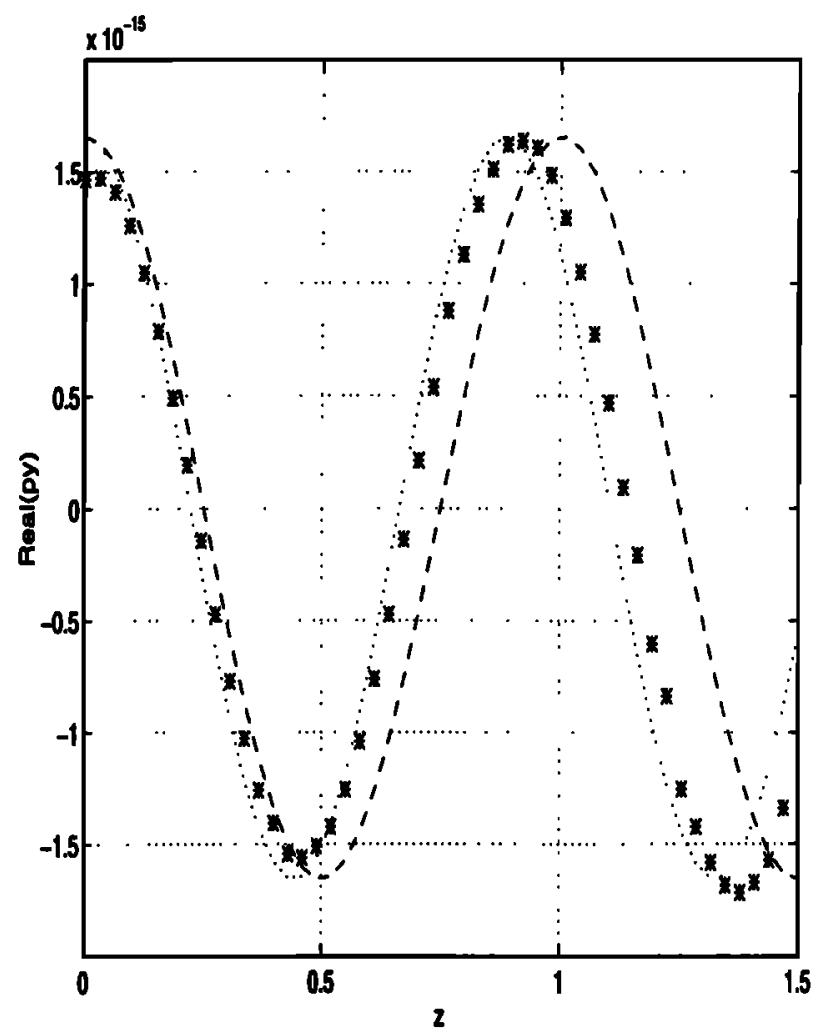

Figure 9. $\operatorname{Re}\left(p_{y}\right)$ versus $z / \lambda_{0}$, where $p_{y}$ is averaged over constant $z$ planes. Monte Carlo results (asterisks) shown for $20 \%$ fractional volume with 5000 spheres with $\epsilon_{s}=3.2 \epsilon_{0}$ in a cubic test volume. Also shown is $\cos \left(K_{\mathrm{r}} z\right)$ for $K_{r}=k_{0} \sqrt{1.27}$ (dotted curve) and $K_{r}=k_{0}$ (dashed curve).

from QCA-CP but were higher than those produced from QCA.

An alternate method of obtaining the real part of the effective permittivity is to plot $\operatorname{Re}\left(p_{y}\right)$ as a function of $z$. The coherent plane wave travels in the $z$ direction through the Monte Carlo cubic volume with a phase determined by $e^{-i K_{r} z_{j}}$. This excites a dipole moment polarized in the $y$ direction whose amplitude traces out a cosine curve with a wavelength of $\lambda_{\text {eff }}=\lambda_{0} / \sqrt{\epsilon_{\mathrm{eff}}}$. The asterisks in Figure 9 represent the average of $\operatorname{Re}\left(p_{y}\right)$ computed along planes orthogonal to the $z$ direction (i.e., $x, y$ planes) for spheres at $35 \%$ fractional volume and with $\epsilon=3.2 \epsilon_{0}$. The dipole oscillation as predicted by the QCA approximation in (27) is shown as a dotted curve 
for $\epsilon_{\text {eff }}=1.27$ and as a dashed curve for free space propagation with $\epsilon_{\text {eff }}=1.00$. The internal dipoles in the Monte Carlo simulation follow closely those predicted by the effective permittivity calculation. There are slight discrepancies close to the ends of the volume (at $z=0$ and $z=1.5 \lambda_{0}$ planes), since these planes are not completely embedded in the effective medium.

\subsection{Active Remote Sensing from a Layer of Snow}

The radiative transfer model can be applied to determine the effect of volume scattering within a layer of discrete spherical scatterers overlying a homogeneous half-space. The results depend on the method in which the scattering coefficient, the phase matrix, and the effective permittivity are determined. In this section we present the results from the classical model, the DMRT model, and the Monte Carlo model. All three models are applied to the geometry shown in Figure 1, where the bottom layer has a permittivity of $\epsilon_{2}=(6.0+i 0.6) \epsilon_{0}$, a typical value for soil. Region 1 is composed of scatterers with $\epsilon_{s}=3.2 \epsilon_{0}$ in a background of air $\epsilon_{1}=\epsilon_{0}$. The scatterers occupy $35 \%$ by volume and have a radius of $0.6 \mathrm{~mm}$ at $16 \mathrm{GHz}$.

Figure 10 shows the backscattering cross section as a function of snow thickness $d$ for an incident angle of $19.31^{\circ}$, as calculated with the three models. In Figure 10 the copolarized backscatter $\sigma_{v v}$ is shown as a dashed curve and the depolarized $\sigma_{h v}$ backscatter as a solid curve. Results from the classical model (top left) have very high copolarized and depolarized levels because of the overestimation of the scattering coefficient from the independent scattering assumption. For the depolarized backscatter the first-order RT solution is zero when the Rayleigh phase matrix is used. The second-order solution is nonzero with $\left|\sigma_{h v}^{(2)}\right| \sim \kappa_{s}^{2} d^{2}$. Thus in the top left and top right plots the copolarized backscatter is a firstorder effect and the depolarization is secondorder. For scatterers at $35 \%$ by volume, DMRT predicts a more reasonable estimate of the scattering coefficient and gives a copolarized level
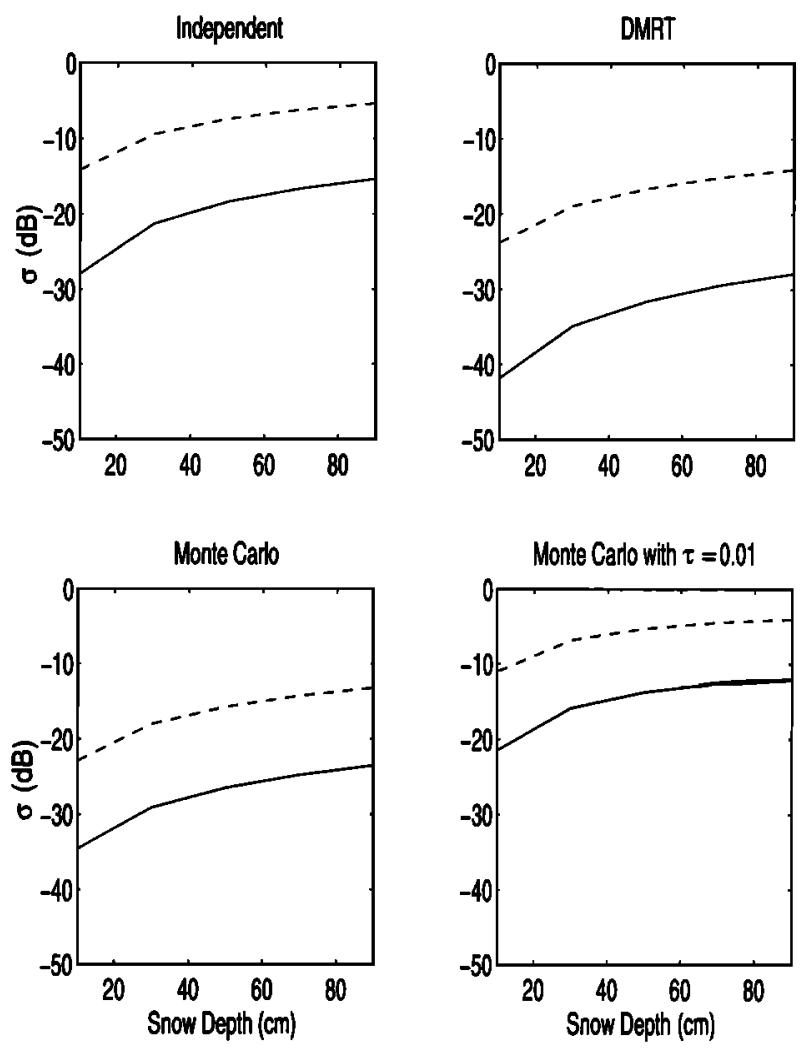

Figure 10. Backscattering as a function of snow depth for $16 \mathrm{GHz}$ at $\theta_{i}=19.31^{\circ}$ from a layer of snow with $a=0.6 \mathrm{~mm}, \epsilon_{s}=3.2 \epsilon_{0}$, and $35 \%$ fractional volume. The dashed curve is $\sigma_{v v}$, and the solid curve is $\sigma_{h v}$. The four plots show results of the radiative transfer theory for the (top left) classical model, (top right) dense media radiative transfer model, (bottom left) Monte Carlo simulations, and (bottom right) sticky particles with $\tau=0.01$.

that is $10 \mathrm{~dB}$ down and a depolarized level that is $13 \mathrm{~dB}$ down relative to the classical model.

The backscattering cross sections from the Monte Carlo model are shown in the bottom two plots. For the nonsticky case (bottom left) the extinction coefficient calculated from the Monte Carlo simulations is not significantly larger than $\kappa_{s}^{\mathrm{DMRT}}$, and the level of the copolarized return is comparable to that from the DMRT model. However, the depolarized backscatter is much greater, particularly at small snow depths such as $10 \mathrm{~cm}$, where it is more than double that predicted by DMRT. This is because the phase matrix from the Monte Carlo simulations has 
nonzero off-diagonal terms, and thus the depolarization has nonzero first-order as well as second-order contributions. The first-order contribution is linearly dependent on $d$ and will therefore be greatest in comparison to the secondorder when the snow depths are small.

In the bottom right plot the backscattering cross sections from the Monte Carlo model are shown when the particles were deposited with a sticking potential of $\tau=0.01$. This scenario could represent snow that has been on the ground long enough for metamorphic forces to cause the grains to become rounded, cluster together, and bond. The effects of this clumping are to increase the effective particle size and thus increase the scattering coefficient to a value approximately 3 times as large as that predicted under the independent scattering assumption for nonsticky particles [Zurk et al., 1995]. Both the copolarized and depolarized backscattering from a layer of clustered snow grains are higher than that calculated under the classical model for nonsticky particles. For these very sticky particles the level of the depolarized backscatter is due mainly to the second-order contributions.

The bistatic cross sections computed using the Monte Carlo model show even greater increases in depolarization relative to conventional theory. Figure 11 shows the bistatic scattering at $\theta_{s}=59^{\circ}$ for spheres with $\tau=0.2$ and the same parameters as the previous plots. The firstorder copolarized return is shown as a dotted curve, the first-order depolarization is shown as a dashed curve, and the second-order depolarization is shown as a dash-dotted curve. The firstorder depolarization, which would be zero under conventional theory, is $7 \mathrm{~dB}$ higher than the second-order at shallow snow depths and reaches $-22 \mathrm{~dB}$ at depths of $90 \mathrm{~cm}$.

The increased extinction predicted from sticky particles can be used to explain the higher levels seen in backscattering data. Results from Monte Carlo simulations of sticky particles are compared with active microwave measurements made on December 16, 1979, at a test site in Colorado [Stiles et al., 1981]. Figure 12 shows the backscattering at $17 \mathrm{GHz}$ for a 47.5 -cm layer of

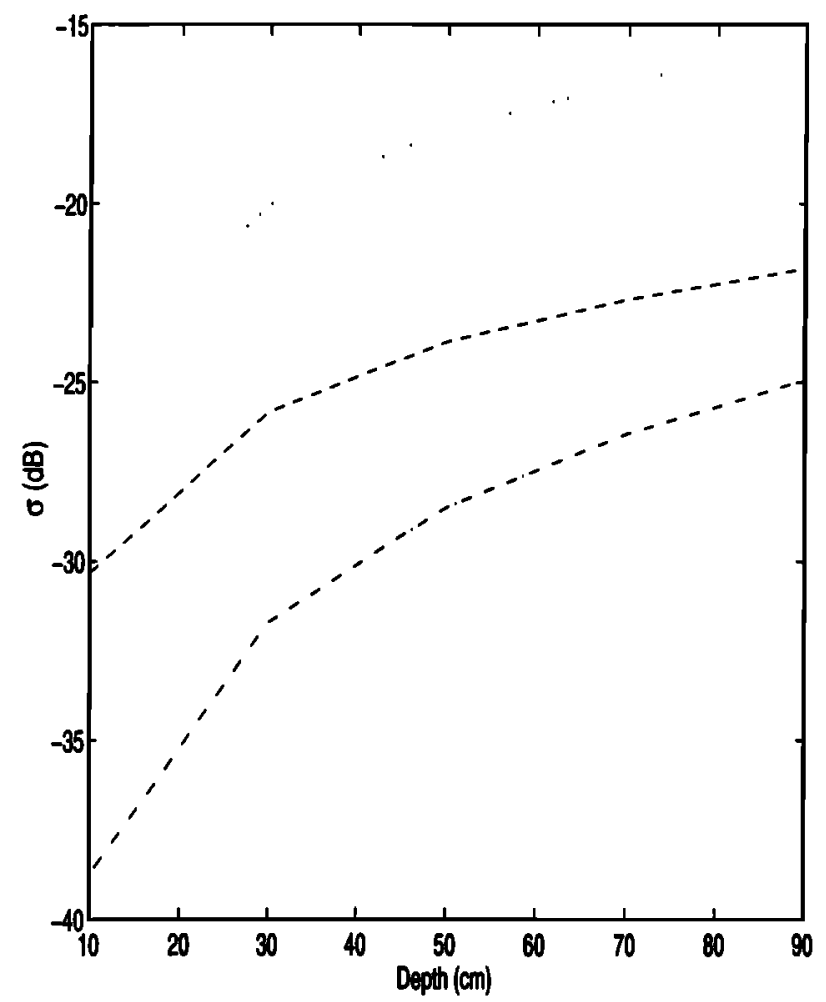

Figure 11. Bistatic cross sections for the same parameters as in Figure 10 except $\theta_{s}=59^{\circ}$ and $\tau=0.20$. The first-order copolarized return is shown as a dotted curve, the first-order depolarization is shown as a dashed curve, and the second-order depolarization is shown as a dash-dotted curve.

snow with grain radius of $a=0.56 \mathrm{~mm}$ and fractional volume of $20 \%$ as a function of incidence angle. The copolarized backscatter (shown as a dotted curve) and the depolarized backscatter (shown as a solid curve) were computed using the extinction coefficient and phase matrix for spheres with $\tau=0.1$.

\section{Discussion and Conclusions}

The Monte Carlo simulations provide a valuable tool for testing our understanding of electromagnetic wave propagation through dense media. The simulations take into account correlated scattering and coherent wave interaction. The coherent and incoherent field distributions can be used to calculate the extinction coefficient, phase matrix, and effective permittivity of 


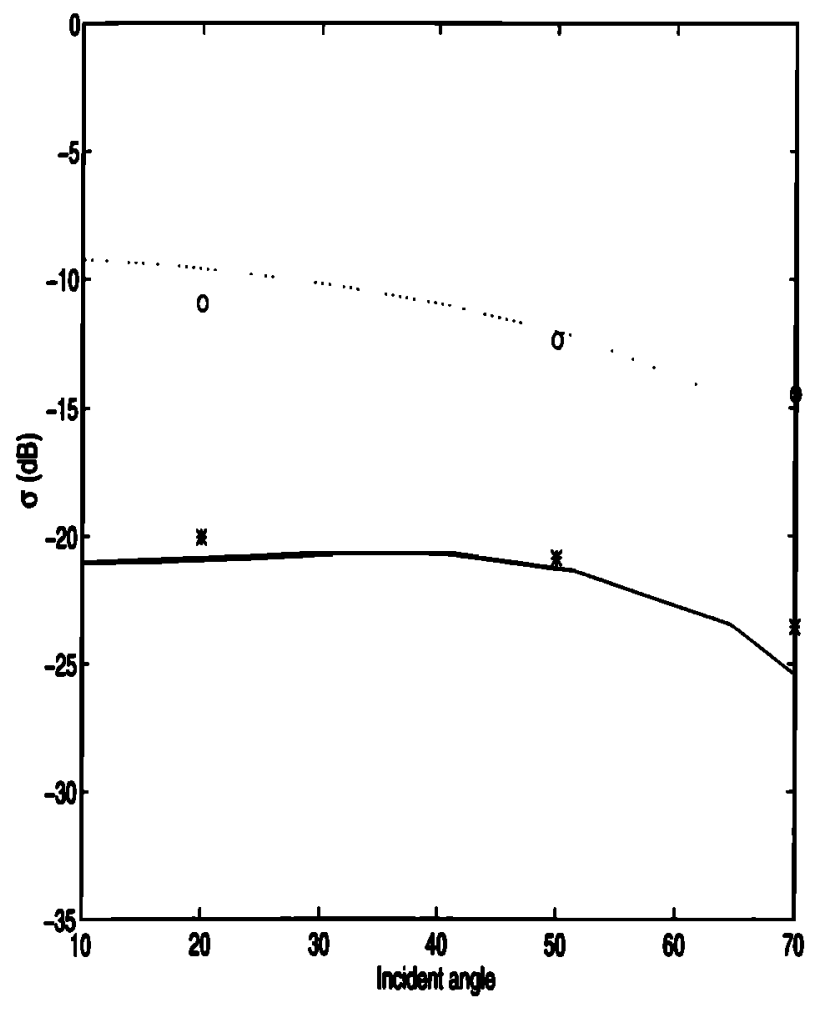

Figure 12. Backscattering cross section for $17 \mathrm{GHz}$ compared with data for a $47.5 \mathrm{~cm}$ deep snow cover with grain radius $a=0.56 \mathrm{~mm}$ and fractional volume $f v=0.2$. Data shown as circles (copol) and asterisks (depol); radiative transfer model with sticky spheres $\tau=0.1$ shown as a dotted curve (copol) and solid curve (depol).

the discrete random medium. These quantities are necessary for solution of the radiative transfer equation. The extinction coefficient from Monte Carlo simulations is significantly lower than that predicted by independent scattering but is comparable with QCA-CP.

The copolarized elements of the phase matrix from the Monte Carlo simulations agree with that obtained from DMRT. However, the offdiagonal elements are nonzero but are 2 orders of magnitude smaller than the copolarized energy. The presence of this depolarization is due to the random organization of the spheres, which can give rise to perturbations in the internal dipole alignment. For very low frequencies the random, isotropic arrangement of spheres causes cancella- tion of these deviations. For higher frequencies, since the wave will only add the dipole moments coherently over scales of the order of $1 / 4$ of a wavelength, the depolarization in the scattered field will remain.

Application of a radiative transfer model can yield different results, depending on the assumptions used in calculation of the extinction coefficient, phase matrix, and effective permittivity. The independent scattering assumption gives excessively high levels of backscatter because of overestimation of scattering. DMRT gives more realistic scattering coefficients but neglects the first-order depolarization effect. First-order depolarization arises from coherent near-field interaction among the spheres that creates nonalignment of dipole moments. Using the phase matrix and scattering coefficient obtained from Monte Carlo simulations can produce copolarized backscattering that is comparable to QCACP but also much higher levels of depolarized backscatter as a result of nonzero contributions from both first- and second-order effects. This combination results in a different snow depth and frequency dependence than indicated by conventional theory. Large increases in both copolarized and depolarized backscatter can be achieved by including the clustered nature of metamorphosed snow in a sticky particle model.

The effective permittivity of snow can be derived from the results of Monte Carlo simulations. The imaginary part of the effective wavenumber is related to the extinction coefficient for the incoherent wave, and the real part corresponds to the phase progression of the coherent wave. The phase information is available both in the coherent scattering from the Monte Carlo volume as well as the oscillation of the internal dipole within the scatterers. The effective permittivity obtained from simulation yields an imaginary part that is in agreement with that predicted by QCA-CP. The real part of the permittivity agrees with that obtained from mixing formulas such as the Clausius-Mossoti formula but is slightly lower than that predicted from QCA-CP. 
Acknowledgments. This work was supported by the office of Naval Research grant N00014-89-J-3132 and the Army Research Office.

\section{References}

Colbeck, S.C., An overview of snow metamorphism, Rev. Geophys., 20(1), 45-61, 1982.

Ding, K. H., L. M. Zurk and L. Tsang, Pair distribution functions and attenuation rates for sticky particles in dense media, J. Electromagn. Waves Appl., 8(12), 1585-1604, 1994.

Ishimaru, A., Wave Propagation and Scattering in Random Media, Academic, San Diego, Calif., 1978.

Ishimaru, A., and Y. Kuga, Attenuation constant of a coherent field in a dense distribution of particles, $J$. Opt. Soc. Am., 72, 1317-1320, 1982.

Koh, G., Experimental study of electromagnetic wave propagation in dense random media, Waves Random Media, 2, 39-48, 1992.

Kuga, Y., F. T. Ulaby, T. F. Haddock, and R. D. DeRoo, Millimeter-wave radar scattering from snow, 1, Radiative transfer model, Radio Sci., 26(2) 329-341, 1991.

Lu, C. C., W. C. Chew, and L. Tsang, The application of recursive aggregate $\mathrm{T}$-matrix algorithm in the Monte Carlo simulations of the extinction rate of random distribution of particles, Radio Sci., 30(1), 25-28, 1995.

Mandt, C. E., Y. Kuga, L. Tsang, and A. Ishimaru, Microwave propagation and scattering in a dense distribution of nontenuous spheres: Experiment and theory, Waves Random Media, 2, 225-234, 1992.

Pak, K., L. Tsang, C. H. Chan, and J. Johnson, Backscattering enhancement of electromagnetic waves from 2-D perfectly conducting random rough surfaces based on Monte Carlo simulations, J. Opt. Soc. Am. A Opt. Image Sci., 12, 2491-2499, 1995.

Shin, R. T., and J. A. Kong, Radiative transfer theory for active remote sensing of a homogeneous layer containing spherical scatterers, J. Appl. Phys., 52, 4221-4230, 1981.

Stiles, W. H., F. T. Ulaby, A. Aslam, and M. Abdelrazik, Active microwave investigation of snowpacks: Experimental documentation, Colorado 1979-1980, RSL Tech. Rep. 410-3, Univ. of Kansas Remote Sens. Lab., 1981.

Tsang, L., and J. A. Kong, Multiple scattering of electromagnetic waves by random distribution of discrete scatterers with coherent potential and quantum me- chanical formulism, J. Appl. Phys., 51, 3465-3485, 1980.

Tsang, L., and J. A. Kong, Effective propagation constants for coherent electromagnetic wave propagation in media embedded with dielectric scatterers, J. Appl. Phys., 53, 716-7173, 1982.

Tsang, L., and J. A. Kong, Scattering of electromagnetic waves from a dense medium consisting of correlated Mie scatterers with size distributions and applications to dry snow, J. Electromagn. Waves Appl., 6(3), 265286, 1992.

Tsang, L., J. A. Kong, and R. T. Shin, Theory of Microwave Remote Sensing, Wiley-Interscience, New York, 1985.

Tsang, L., C. Mandt, and K. H. Ding, Monte Carlo simulations of the extinction rate of dense media with randomly distributed dielectric spheres based on solution of Maxwell's equations, Opt. Lett., 17(5), 314-316, 1992.

Varadan, V., and V. V. Varadan, Multiple scattering of electromagnetic waves by randomly distributed and oriented dielectric scatterers, Phys. Rev. D. Part Fields, 21 (8), 388-394, 1980.

Varadan, V., V. N. Bringi, and V. V. Varadan, Coherent electromagnetic wave propagation through randomly distributed dielectric scatterers, Phys. Rev. D. Part Fields, 19, 2480-2489, 1979.

West, R., D. Gibbs, L. Tsang, and A. K. Fung, Comparison of optical scattering experiments and the quasicrystalline approximation for dense media, J. Opt. Soc. Am. A Opt. Image Sci., 11, 1854-1858, 1994.

Zurk, L. M., L. Tsang, K. H. Ding, and D. P. Winebrenner, Monte Carlo simulations of the extinction rate of densely packed spheres with clustered and nonclustered geometries, J. Opt. Soc. Amer. A Opt. Image Sci., 12, 1771-1781, 1995.

L. Tsang, Electrical Engineering Department, University of Washington, Seattle, WA 98195. (e-mail: tsang@ee.washington.edu)

D. P. Winebrenner, Applied Physics Laboratory, University of Washington, Seattle, WA 98195. (e-mail: dpw@apl.washington.edu)

L. M. Zurk, MIT/Lincoln Laboratory, 224 Wood Street, Lexington, MA 02173. (e-mail: zurk@ll.mit.edu)

(Received August 11, 1995; revised March 4, 1996; accepted March 6, 1996.) 